\title{
Mobile Application of Electronic Prescribing for Supporting E- Health Services
}

\author{
M. Miftakul Amin ${ }^{1, *}$, Adi Sutrisman ${ }^{1}$, Deris Stiawan', and Ermatita ${ }^{2}$ \\ ${ }^{1}$ Department of Computer Engineering, Politeknik Negeri Sriwijaya, Palembang - Indonesia \\ ${ }^{2}$ Department of Computer Engineering, Sriwijaya University, Palembang - Indonesia
}

\begin{abstract}
The frequency of frequent medication errors in misreading prescriptions and drug doses that have the potential to cause harm and endanger the patient's health can be reduced by building electronic prescription applications (e-prescribing). The purpose of the development of e-prescribing is that prescription data that has been previously given to patients, can be traced to the history of prescription administration. A patient can seek treatment at more than one health provider organization so that prescription data provided by each health provider can ideally be traced and accessed through smartphone devices that are currently widely used by patients. In this research, a mobile application for electronic prescription has been built using RESTful Web Service as a WEB API that is used to integrate various prescription data from health providers. On the client-side mobile applications are used to access electronic prescription services. This research has provided an alternative prescription with information retrieval that is faster, more economical in the use of paper media, and integration of health information can be done in a framework so that it will improve health services to patients.
\end{abstract}

Keywords: E-Prescribing; RESTful Web Service; Mobile Application; E-Health Service.

\section{Introduction}

The use of smartphones in several developing countries has a significant increase. This is characterized by increasing interaction between smartphone users and various applications and services provided by developers, both by government and non-government organizations.

Smartphone has significant impact on consumer and their lifestyle because the phones can work as small computer [1]. On the other hand, there are still few services in the health sector that can bridge access to health information for smartphone users [2]. Many efforts have been made to realize services in the health sector including presenting Personal Health Record (PHR) data [3], Treatment and appointment, clinical data, and financial, which is commonly known as Mobile Health $[4,5,6,7]$, although some of these systems are not connected to each other [8].

In the development of the world of health in Indonesia, drug prescription systems are still experiencing many obstacles such as errors in interpreting handwritten prescription drugs by doctors, mistake in determining drug dosage and spend a longtime getting medicine. Besides that, the objective conditions that occur are that patients carry out medical examinations and get prescription drugs from several health care organizations that are seen from the history of prescribing, the data will be stored in each of these organizations.
A common problem that often occurs in pharmaceutical installations in a health organizing organization such as hospitals, health clinics, and health centers is the occurrence of human error and medication errors. Human error is characterized by errors in prescribing data, while medication errors occur due to incorrect prescribing to patients that can harm and endanger the health of patients. The Institute of Medicine (IOM) provide information that $32-69 \%$ from medication error is a preventable event [9]. Reports from several countries show that medication errors have a large impact on mortality, morbidity, and increased maintenance costs.

Giving prescription drugs is a very important element for patients, so the patient's dependence on therapy for healing diseases is very high. For health care organizations, excessive workload, staff shortages, and fatigue are often factor in human error in drug administration. To overcome this, innovation is needed so that errors in drug administration can be minimized.

\section{Literature Review}

\subsection{Electronic Prescribing}

According to the description of Centers for Medicare \& Medicaid Services stated that e-prescribing was initiated by five health institutions in America that were connected through electronic health (E-health). e-

\footnotetext{
* Corresponding author: miftakul a@polsri.ac.id
} 
prescribing interpreted as a recipe that has been transmitted using electronic media that can connect several or various information between doctors, pharmacies, and financial departments, in health plans both directly and indirectly [10]. E-Prescribing is also defined as a computer system used to produce recipes electronically, not written using paper [11].

\subsection{Service Oriented Architecture}

Service Oriented Architecture (SOA) is an architecture methodology and design for implementing in distributed system [12]. It involves three main parts, namely service providers, service consumers, and service broker [13]. It provides as set of interfaces to enable clients to consume the services [14]. The web services are the main component of SOA Architecture [15].

The implementation of SOA in the health sector involves data packaged in a variety of data formats both structured and unstructured [16], and stored in various databases that are relational databases, file servers, or noSQL database.

\subsection{RESTful Web Services}

Web services are one technology that can be used to perform data integration between information systems [17]. RESTful Web services are marked by accessing an unique URI (Uniform Resource Locator) address and using HTTP method such as GET, POST, PUT, DELETE [18, 19]. Some of these HTTP methods equivalent to CRUD (Create, Read, Update, Delete) operation owned by the Database Management System as shown in Table 1. Resources that can be accessed by RESTful web services can be hypertext, files, or scripts [20]. Web services allow merging or composition of several web services to be a framework that has a certain performance [21].

Table 1. HTTP Method and CRUD Operation.

\begin{tabular}{|c|c|}
\hline HTTP Method & CRUD Action \\
\hline GET & Retrieve a resource \\
\hline POST & Create a resource \\
\hline PUT & Update a resource \\
\hline DELETE & Delete a resource \\
\hline
\end{tabular}

In implementing web services using mobile applications, programmers can deploy, discover, publish, and execute web services using wireless medium [22] and networking technology support also peer to peer interaction [23]. Various services that will be accessed through mobile applications can also be placed in the cloud making it easier to access with high mobility [24].

\section{Methodology}

\subsection{Architectural Framework for E-Prescription System}

In this study using the system architecture model as shown in Fig. 1, there is a RESTful web service in which there are services in the form of several methods that can be run remotely. To run the service, the client application uses the HTTP Method as seen in Table 1.

In the REST architecture (REpresentational State Transfer) web services generally use the HTTP (Hypertext Transfer Protocol) protocol to communicate data. In this REST architecture, the REST server provides resources (resources / data) and the REST client accesses and displays these resources for future use. Each resource is identified by URIs (Universal Resource Identifiers) or global ID. These resources are represented in text format, JSON or XML.

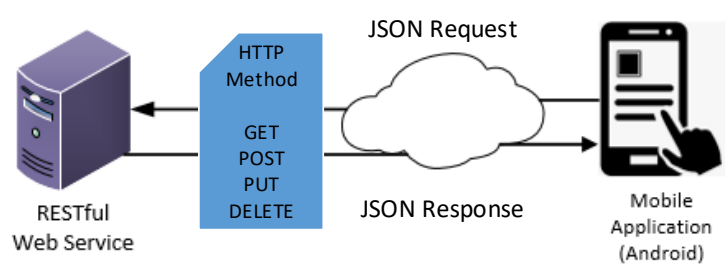

Fig. 1. The System Process Diagram

This e-prescribing mobile application was built using the Android platform, through the mechanism of Request / Response data exchanged in JSON format.

\subsection{The System Process Diagram}

A series of processes that exist in a mobile e-prescribing application can be seen in Fig. 2. The system provides services for users to register users before logging in to the system. Furthermore, when the login validation of the user is successful, the system will display a number of menus which, if selected, will display information according to the requested functions such as user profile data, view recipe, view HPO, change password and sign out. Users can end interacting with the system, which is enough to select the signout menu that will bring the user back to the register/login menu. 


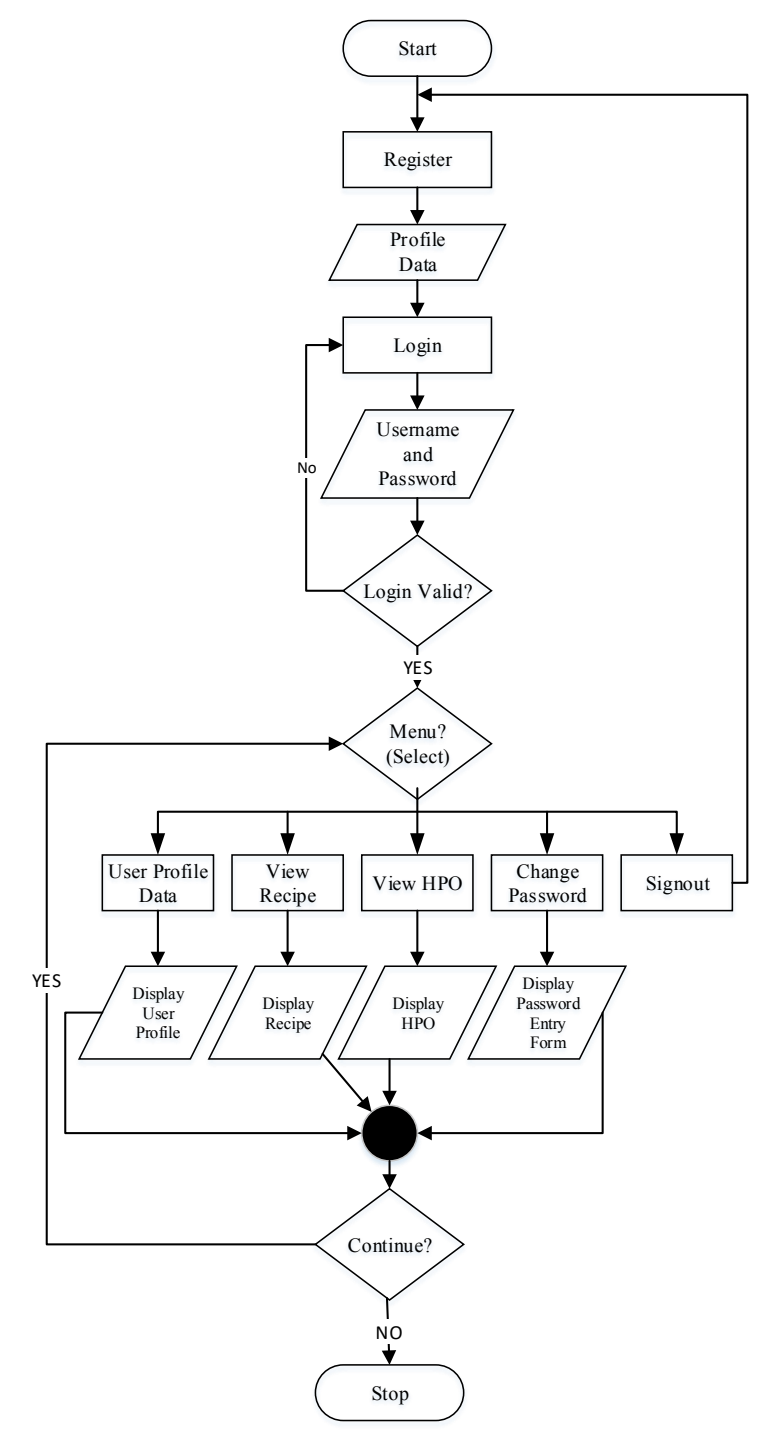

Fig. 2. The System Process Diagram

\section{Results and Discussion}

\subsection{Mobile Application System}

The mobile application of e-prescribing system is tested using emulators of Android Development Tools from Google and several real device smartphones. Fig. 3 shows the first time a mobile application is loaded in smartphone memory. In this view, the patient acts as a user can log in by entering a user id and password, but if you do not have an account to log in, you can register first by entering some information needed by the system. Fig. 4 shows the menu display to improve user profile data for active users. This menu provides a number of data items that can be filled directly to improve existing data. In addition, the system also provides a list of health organization providers that are part of the system. The Fig. 5 there is a menu display for changing passwords for active users, as well as a list of recipes that have been given to patients from various health organization providers. This information is a history of a number of recipes that have been given to patients.

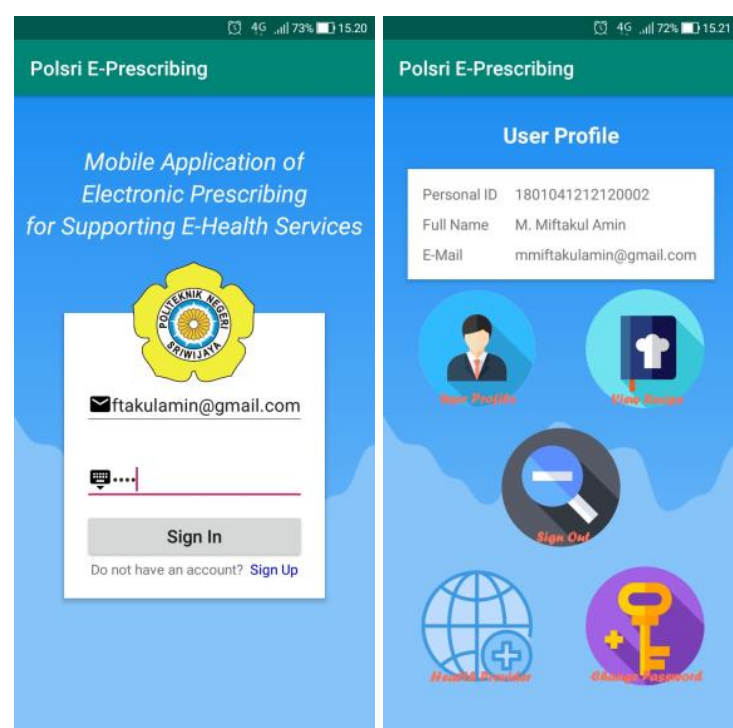

Fig. 3. Mobile Application Sig in/Sign up and Home Menu
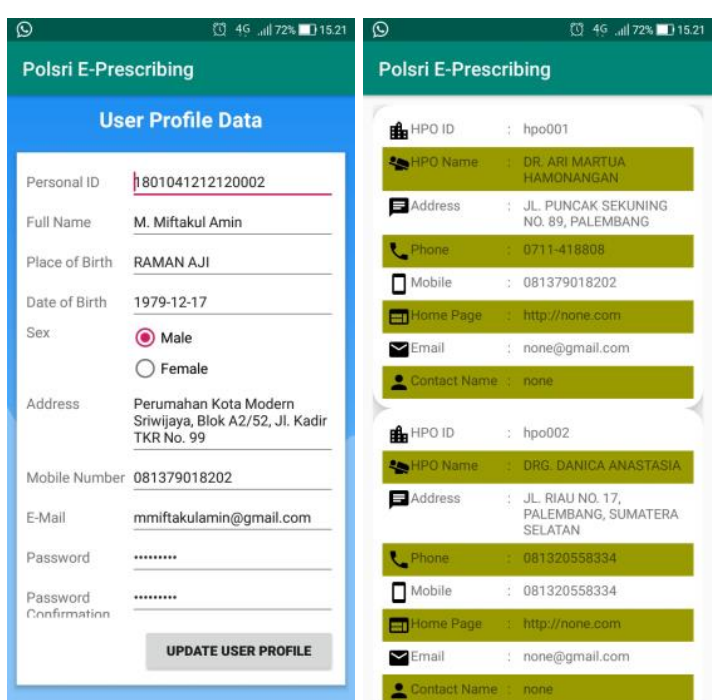

Fig. 4. User Profile and Health Provider Organization Menu

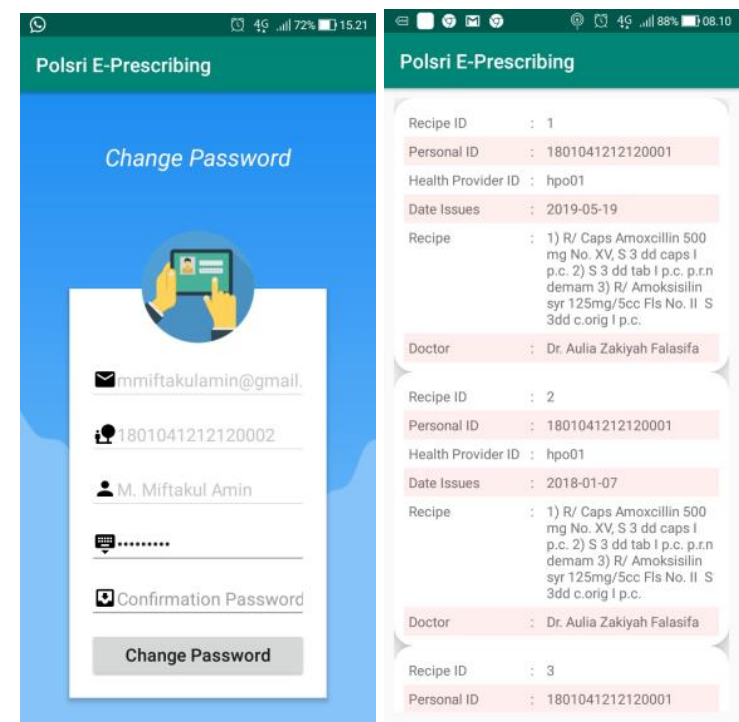

Fig. 5. Change Password and List of Recipe Menu 


\subsection{Performance of System}

Table 2 shows the performance of the method execution owned by RESTful web services. Tests are carried out both on local servers, and online. The response speed given by the server in milliseconds shows that execution on the local server is faster than the execution on the online server. The execution values can be different in the trial session because they are influenced by network bandwidth and payload.

Table 2. RESTful Web Services Performance

\begin{tabular}{|c|c|c|c|}
\hline No. & $\begin{array}{c}\text { Name of } \\
\text { Services }\end{array}$ & $\begin{array}{c}\text { Local } \\
(\mathrm{ms})\end{array}$ & $\begin{array}{c}\text { Online } \\
(\mathrm{ms})\end{array}$ \\
\hline 1. & cekEmail & 10 & 80 \\
\hline 2. & changePassword & 41 & 92 \\
\hline 3. & getHPO & 26 & 92 \\
\hline 4. & getRecipe & 14 & 74 \\
\hline 5. & getUser & 8 & 97 \\
\hline 6. & getUserByEmail & 18 & 112 \\
\hline 7. & getUserByPin & 17 & 122 \\
\hline 8. & signin & 38 & 92 \\
\hline 9. & signup & 42 & 100 \\
\hline 10. & updateUser & 23 & 121 \\
\hline
\end{tabular}

Figure 6 presents information graph average method execution provided by RESTful web services. These data are taken from the execution process using POSTMAN software to determine the speed and amount of data transmitted between client/server and JSON data format.

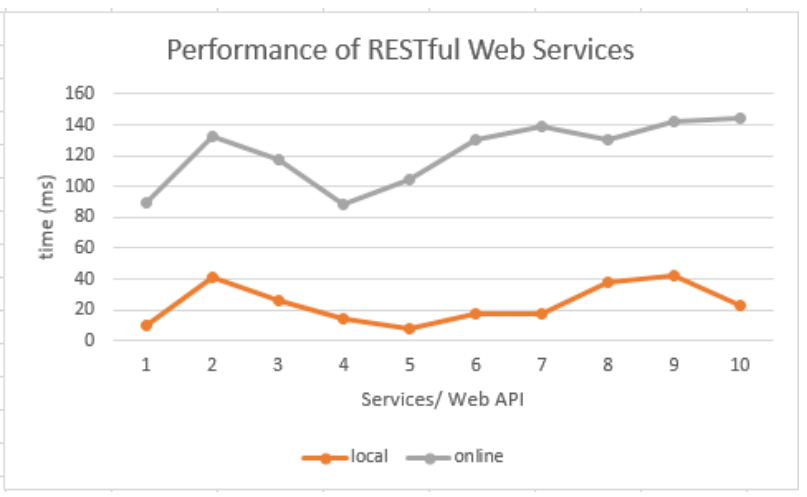

Fig. 6. Processing Graph Services

Test image in Figure. 6 is performed on a local server or a remote server that is specified on the internet service provider web server. From the graph, it is seen that the web service execution performed on the local server and hosting server shows the consistency response time. The graph also shows execution on the local server showing a faster response time compared to execution on the hosting server. Regarding speed, response time is determined by computing resources, bandwidth, and the payload or content of transmitted data.

\section{Conclusion}

Based on the results and discussion that has been done, it can be concluded some conclusions: this mobile prescribing application can integrate prescription information from several health providers so that the history of prescribing information can be traced and accessed in full. This can reduce the occurrence of medication errors for patients. RESTful Web Service can be used as a bridge to integrating data and information from several different health providers, so that information can be accessed easily.

\section{References}

1. V.S. Rao, and T.M. Krishna, A Design of Mobile Health for Android Applications, American Journal of Engineering Research (AJER), 3, 6: 20-29, (2014)

2. E. Eze, R. Gleasure, and C. Heavin, Reviewing mHealth in developing countries: A stakeholder perspective, Procedia Computer Science, 100: 10241032 (2016)

3. M.A. Dahdah, A.D. Du Lou, and C. Meadel, Mobile health and maternal care: A winning combination for healthcare in the developing world?, Health Policy and Technology, 4: 225-231 (2015).

4. C. Free, G. Phillips, L. Watson, L. Galli, L. Felix, P. Edwards, V. Patel, and A. Haines, The Effectiveness of Mobile-Health Technologies to Improve Health Care Service Delivery Processes: A Systematic Review and Meta-Analysis, PLOS Medicine, 10, 1: 1-26, (2013).

5. A. Ekiyor, and M.A.S.G. Yalkin, Mobile Health Applications with Smartphones, International Journal of Management and Applied Sciences, 2, 9: 140-143 (2016).

6. C. Free, G. Phillips, L. Felix, L. Galli, V. Patel, and P. Edwards, The Effectiveness of M-health technologies for improving health and healt services: a systematic review protocol, IBM Research Notes, 3: 250 (2010).

7. V.K. Minimol, and R.S. Shaji, M-Health Framework for Public Health Services, International Journal of Applied Engineering Research, 12, 20: 10164 - 10168 (2017).

8. F.Chali, Z.O. Yonah, and K. Kalegele, Data Exchange Architecture for the Development of Mobile Applications that Support eHealth Systems Interoperability: A Case of Tanzania, International 
Journal of Advanced Computer Research, 8, 34: 110 (2017).

9. L. Kohn, J. Korrigan, and M. Donaldson, To Err is Human: Building a Safer Health System, Edition. National Academy Press Washington DC, (2000).

10. C.F.M. \& M. Services, e-prescribing, USA: http://www.cms.gov, (2019).

11. O.M.A. Adlan, and A.J. Alzubaidi, Electronic Prescribing Design (E-Prescribing), IOSR Journal of Engineering (IOSRJEN), 05, 02: 24-27 (2015).

12. M.M. Amin, A.D.I, Sutrisman, D.Stiawan, Ermatita, and A. Maseleno, Design restful web service of national population database for supporting ehealth interoperability service, Journal of Theoretical and Applied Information Technology, 96, 15: 4794-4805 (2018).

13. S.K. Nahak, D.P. Mohapatra, M.R. Patra, Survey on Service Oriented Architecture Testing, International Journal of Advance Computing Techniques and Applications, 2, 2: 102-105 (2014).

14. A.A. Al-Rashedi, E-Government Based on Cloud Computing and Service Oriented Architecture, International Journal of Computer and Electrical Engineering, 6, 3: 201-206, (2014).

15. O. Dospinescu, and M. Perca, Web Services in Mobile Application, Informatica Economica, 17, 2: 17-26, (2013).

16. A.A. Lahoti, and P.L. Ramteke, Advanced Healthcare System using E-Health \& M-Health in Cloud \& Mobile Environments, International Journal of Engineering Sciences \& Research Technology, 4, 2: 544-550 (2015).

17. W.H. Utomo, B2B Integration based on SOA Using Web Service, International Journal of Computer Applications, 32, 2: 41-48 (2011).

18. A.S. Johal, and B. Singh, Performance Analysis of Web Services for Android based Devices, International Journal of Computer Application, 92, 11: 43-46 (2014).

19. K. Elgazzar, and P. Martin, Mobile Web Services: State of the Art and Challenges, International Journal of Advanced Computer Science and Applications, 5, 3: 173-188 (2014).

20. A. Dudhe, and S.S. Sherekar, Performance Analysis of SOAP and RESTful Mobile Web Services in Cloud Environment, International Journal of Computer Applications, pp. 1-4, (2014).

21. P. Thangaraj, P. Balasubramanie, D. Jugisathvi, and S.A. Bhuvanasoundarya, A Prototype for Composing SOAP, RESTful and Mobile Web Services, International Journal of Scientific \& Engineering Research, 7, 2: 55-59 (2016).

22. A. Dudhe, and S.S. Sherekar, Exploring Mobile Web Service Framework, International Journal of Innovations \& Advancement in Computer Science, 6, 2: 285-289 (2017).

23. P. Prabhavathy, S. Bose, A. Kannan, and C. Gopinath, Smart Phone User Assistance Application for Android, Journal of Engineering Science and Technology, 10, 9: 1203-1214 (2015).

24. D.K. Kim, Development of Mobile Cloud Applications using UML, International Journal of Electrical and Computer Engineering (IJECE), 8, 1: 596-604 (2018). 